\title{
Hydrodynamic simulations of irradiated secondaries in dwarf novae
}

\author{
M. Viallet and J.-M. Hameury \\ Observatoire Astronomique, Université Louis Pasteur and CNRS, 11 rue de l’Université, 67000 Strasbourg, France \\ e-mail: [viallet; hameury]@astro.u-strasbg.fr
}

Received 4 May 2007 / Accepted 21 August 2007

\begin{abstract}
Context. Secondary stars in dwarf novae are strongly irradiated during outbursts. It has been argued that this could result in an enhancement of the mass transfer rate even though the $L_{1}$ region is shaded from the primary irradiation by the accretion disc. Previous investigations of the possibility of a circulation flow transporting heat from hot regions to $L_{1}$ have given opposite answers.

Aims. We investigate the surface flow of irradiated secondaries numerically. We consider the full time-dependent problem and take the two-dimensional nature of the flow into account.

Methods. We use a simple model for the irradiation and the geometry of the secondary star: the irradiation temperature is treated as a free parameter and the secondary is replaced by a spherical star with a space-dependent Coriolis force that mimics the effect of the Roche geometry. The Euler equations are solved in spherical coordinates with the TVD-MacCormack scheme.

Results. We show that the Coriolis force leads to the formation of a circulation flow from the high-latitude region to the close vicinity of the $L_{1}$ point. However, no heat can be efficiently transported to the $L_{1}$ region due to the rapid radiative cooling of the hot material as it enters the equatorial belt shaded from irradiation. Under the assumption of hydrostatic equilibrium, the Coriolis force could lead to a moderate increase in the mass transfer by pushing the gas in the vertical direction in the vicinity of $L_{1}$, but only during the initial phases of the outburst (about 15-20 orbital periods). It remains possible, however, that this assumption breaks up due to the strong surface velocity of the flow transiting by $L_{1}$, close to the sound speed. In this case, however, a three-dimensional approach would then be needed to determine the mass flux leaving the secondary.

Conclusions. We therefore conclude that the Coriolis force does not prevent a flow from the heated regions of the secondary towards the $L_{1}$ region, at least during the initial phases of an outburst, but the resulting increase in the mass transfer rate is moderate, so unlikely to be able to account for the duration of long outbursts.
\end{abstract}

Key words. accretion, accretion disks - stars: binaries: close - stars: novae, cataclysmic variables - stars: dwarf novae

\section{Introduction}

Dwarf novae (DN) are cataclysmic variables that undergo outbursts, i.e. a sudden increase in their luminosity by a few magnitudes lasting for a few days (see e.g. Warner 1995). It is now widely believed that these outbursts are due to a thermal/viscous instability of the disc triggered when hydrogen becomes partially ionized. In the standard model of DN (often referred to as the DIM, see Cannizzo 1993; Lasota 2001, for reviews), the unstable accretion disc performs a limit cycle between an outburst phase with a high accretion rate onto the compact object and a quiescence phase with a low accretion rate where the disc rebuilds. The role of the irradiation of the secondary star during an outburst is still a matter of debate.

During an outburst, the secondary is likely to be significantly affected by the strong increase in the accretion luminosity emitted by the primary, as the irradiation flux may exceed the intrinsic stellar flux of the secondary by a large amount (see Smak 2004a). It has often been argued, but not yet demonstrated, that this could result in a mass transfer enhancement that could, if significant, be an essential ingredient of the DIM. For example, it has been suggested that irradiation-induced mass transfer enhancement is the origin of outburst bimodality (Smak 1999) or that it could play an important role in the outburst/superoutburst phenomenon of the SU UMa stars (see Hameury 2000; Hameury et al. 2000; Smak 2000).
However, the $L_{1}$ point is shaded by the accretion disk (Sarna 1990) and thus is not under the direct influence of irradiation. The very existence of a circulation flow transporting heated matter from the irradiated region toward the Lagrange point is not clear, and it is not known if this results in a substantial increase in the mass transfer rate.

There are some observational clues (see Vogt 1983; Smak 1995; Smak 2004a) that the mass transfer rate in some systems is increased during an outburst or a superoutburst. These observational arguments have been questioned, however, by Osaki \& Meyer (2003), who use theoretical grounds to argue against the possibility of such a mass transfer enhancement. First, we do not know how the accretion flux interacts with the secondary envelope, and it is not known if irradiation can significantly raise the effective temperature on the secondary. Second, even in the case where high temperature gradients and therefore high pressure gradients are raised, Osaki \& Meyer (2003) argue that the strong Coriolis force prevents the formation of a circulation flow transporting heat toward $L_{1}$.

Their main argument is that the Coriolis force remains significant near $L_{1}$, due to the particular shape of the Roche lobe (contrary to the spherical case where the Coriolis force vanishes at the equator). Its magnitude remains large enough to deflect any meridional flow to what is called a geostrophic flow, a steady state where velocity field lines are parallel to isobars (see Pedlosky 1982). These conclusions were challenged, 
Table 1. Physical parameters for the three cases investigated in this paper.

\begin{tabular}{lccccccccc}
\hline \hline System & Model number & $P_{\text {orb }}(\mathrm{h})$ & $a\left(R_{\odot}\right)$ & $R_{2} / a$ & $q=M_{2} / M_{1}$ & $T_{\star}(\mathrm{K})$ & $T_{\text {irr }}(\mathrm{K})$ & $\Sigma_{\text {irr }}\left(\mathrm{g} \mathrm{cm}^{-2}\right)$ & Shadow height \\
\hline OY Car & 1 & 1.51 & 0.6 & 0.21 & 0.1 & 2500 & 10000 & 350 & $10^{\circ}$ \\
U Gem & 2 & 4.24 & 1.48 & 0.29 & 0.36 & 3500 & 10000 & 200 & $10^{\circ}$ \\
Z Cam & 3 & 6.96 & 2.17 & 0.35 & 0.6 & 4200 & 10000 & 250 & $10^{\circ}$ \\
\hline
\end{tabular}

$P_{\text {orb }}, a, q$ have their usual meanings. $R_{2}$ is the mean-radius of the secondary. The secondary surface temperature $T_{\star}$ is taken from Smak (2004a). $T_{\mathrm{irr}, 0}$ is the maximum irradiation temperature reached during an outburst, $\Sigma_{\mathrm{irr}}$ is the column density of the irradiated layer when $T=T_{\mathrm{irr}}$. The last column is the height of the shadow boundary above the equator when measured on the main meridian.

nevertheless, by computations by Smak (2004a), showing streamlines converging to the $L_{1}$ region. In subsequent papers, Osaki \& Meyer (2004) and Smak (2004b,c) developed their argumentation without reaching any agreement.

We must point out that the geometric argument from Osaki $\&$ Meyer is a 1D argument, valid only on the $L_{1}$ meridian. As the $L_{1}$ point is a singular point in the Roche geometry, the strength of the Coriolis force changes very rapidly as one moves away from $L_{1}$ on the equator; this leaves open the possibility that the heated material does not follow the shortest way along the meridian, but that it follows an "easier" way to reach the $L_{1}$ region. Smak (2004a) computations were done in a 2D steady state and thus take this effect into account. The main flaw of both approaches is that the time scale to reach a steady state could be long, possibly longer than the duration of an outburst or that such a steady state could never be reached.

We investigate the dynamics of the irradiated envelope here by using $2 \mathrm{D}$ hydrodynamic simulations. We adopt a very simple model for the irradiation and geometry of the secondary, but, unlike Smak (2004a), we solve the full time-dependent problem. Our numerical simulations show that the flow can indeed reach the vicinity of the $L_{1}$ point thanks to (and not despite) the Coriolis force. However, as hot material crosses the boundary between regions directly illuminated by the primary star and regions shaded by the accretion disc, it cools down so rapidly that any significant heating of the $L_{1}$ point cannot be obtained. If vertical hydrostatic prevails, no mass transfer enhancement can occur. However, velocity magnitudes close to the sound speed are obtained in the vicinity of the $L_{1}$ point, so strong vertical velocity gradients are expected, which could lead to turbulence and/or the breakdown of hydrostatic equilibrium assumption. In this case, one would need a full 3D analysis of the problem, far beyond the scope of this paper.

The paper is organized as follow. We present the model in Sect. 2 and the numerical code in Sect. 3. Our results are given in Sect. 4, and we summarize our conclusions in Sect. 5.

\section{The model}

We consider three models with characteristics of the three DN subtypes. The main physical parameters used are given in Table 1.

\subsection{Geometrical considerations for the dynamics}

A complete investigation of the problem would require $3 \mathrm{D}$ radiative hydrodynamic simulations of the stellar envelope in the full Roche potential. The problem has to be simplified to be tractable. As the scale height of the atmosphere is small compared to the stellar radius, $h / R \ll 1$ (even in the vicinity of the $L_{1}$ point), the vertical dynamical time scale is small compared to all other time scales, and the vertical hydrostatic equilibrium should be

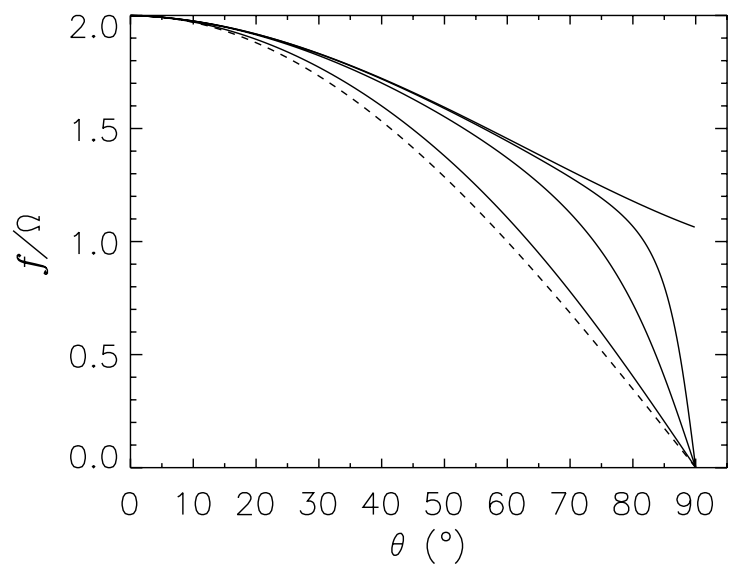

Fig. 1. Profile of the Coriolis parameter $f$ along different meridians of the Roche lobe: $\phi=0^{\circ}\left(L_{1}\right.$ meridian $), \phi=5^{\circ}, \phi=15^{\circ}$ and $\phi=45^{\circ}$ (from top to bottom). For comparison the profile corresponding to spherical geometry is also shown in dashed line.

reached rapidly. A 2D approach should be sufficient for investigating the important features of the surface flow (see however the discussion in Sect. 4.3 and in the conclusion).

We therefore solve the full set of the Euler equations on the surface of the secondary star, whose surface is a Roche equipotential. As these systems have short orbital periods, the Coriolis force is an essential dynamical ingredient. In 2D the Coriolis force only enters the equations via its component parallel to the surface. Its strength is characterized by the Coriolis parameter $f=2 \Omega . n$ where $\boldsymbol{n}$ is the local normal to the surface. Figure 1 shows the profile of $f$ along different meridians of the Roche lobe. On the $L_{1}$ meridian, it can be seen that the Coriolis parameter remains large as stated in Osaki \& Meyer (2003, 2004), but the $\theta$ dependence of $f$ becomes more and more similar to the spherical case as one moves away from $L_{1}$. Figure 2 shows the strong dependence of $f$ on longitude, showing that a $1 \mathrm{D}$ calculation is insufficient.

The ratio of the inertial force to the Coriolis force is the Rossby number:

$\Re_{0}=\frac{V}{f L}$,

where $V$ and $L$ are characteristic values of the velocity and length. In our case the relevant scales are $L \sim R_{2}$, the meanradius of the secondary, $V \sim c_{\mathrm{s}}$, the sound speed, and $f \sim \Omega$, yielding $\Re_{\mathfrak{D}} \sim 0.04$ for all models (see Table 1 ). As expected, the Coriolis force dominates the dynamics on the secondary.

One should in principle use Roche coordinates (see Kopal 1969) to describe the secondary surface. For simplicity, we consider a spherical star of radius $R_{2}$ (the mean radius of the Roche lobe) with a space-dependent Coriolis parameter that mimics the Roche geometry. This simplifies the problem, as spherical coordinates are much simpler to use than Roche coordinates. The 


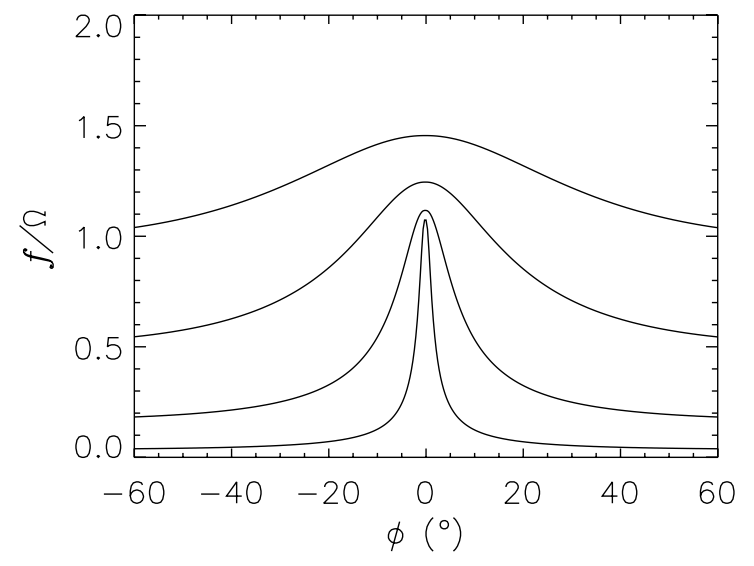

Fig. 2. Profile of the Coriolis parameter $f$ on the Roche lobe at colatitudes $\theta=89^{\circ}, \theta=85^{\circ}, \theta=75^{\circ}$, and $\theta=60^{\circ}$ (from bottom to top).

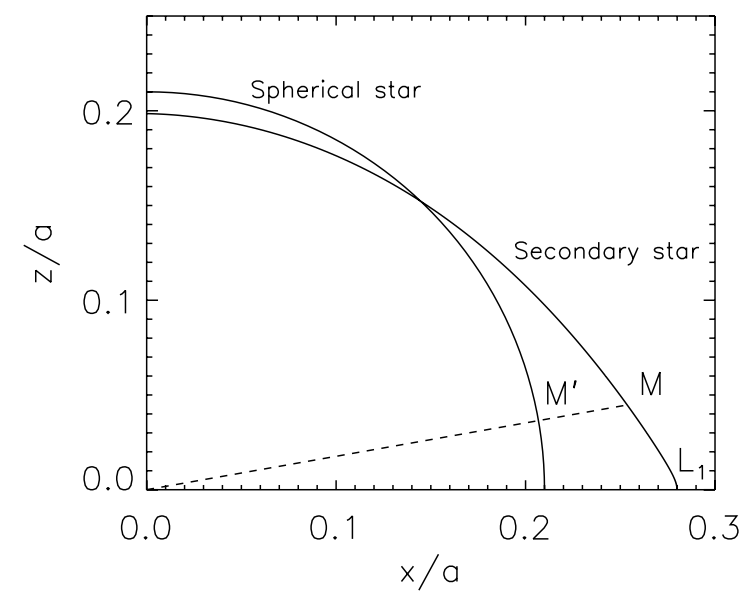

Fig. 3. Map $M \rightarrow M^{\prime}$ from the Roche equipotential to the sphere of radius $R_{2}$ (the mass ratio is $q=0.1$ ). The figure shows a cut along the $L_{1}$ meridian.

mapping between the Roche equipotential and the sphere is illustrated in Fig. 3; we use a Cartesian system of coordinates where the center of mass of the secondary is at the origin, the center of mass of the primary is at $(a, 0,0)$ with $a$ the orbital separation, and the $z$ axis is perpendicular to the orbital plane. The spherical approximation is poor in the $L_{1}$ region, where departure from spherical geometry is the most important. This has two consequences. First the geometrical terms in the Euler momentum equation are not taken into account very well. However, in this region, the dynamic is dominated by the Coriolis force rather than by geometrical effect (note that the Rossby number is also the ratio of geometric terms to the Coriolis one in the Euler equations). Second we underestimate distances: for example the distance from the shadow boundary to $L_{1}$ is underestimated by $\sim 20-30 \%$ in our model (see Fig. 3). This is not a serious difficulty, since it is easy to extrapolate our results to a real Roche geometry.

The Roche geometry is singular at $L_{1}$, but we assume that the stellar surface is at a minimum distance $\Delta r=0.02 R_{2}$ below $L_{1}$, comparable to the vertical scale height of the atmosphere. With this assumption, $f(\theta, \phi)$ is no longer singular, as was the case in Fig. 1, but instead drops sharply for $\theta \gtrsim 85^{\circ}$ and vanishes exactly for $\theta=90^{\circ}$ along the $L_{1}$ meridian $\left(\phi=0^{\circ}\right)$. The resulting $f$ profile does not depend much on $q$ (the binary mass ratio) and we take here $q=0.1$.

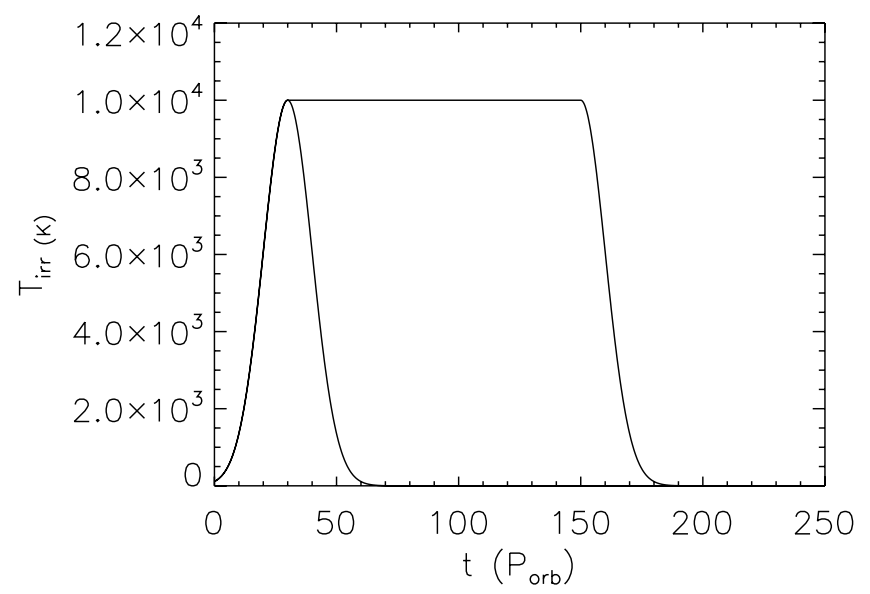

Fig. 4. Time profiles of normal outburst and of superoutburst. The superoutburst profile has the same rise and fall but has a long plateau at maximum.

\subsection{Irradiation}

It is still being debated whether the accretion flux can significantly heat the secondary atmosphere, we leave this problem to a future investigation. Here we model the irradiation of the secondary in a crude, but simple way: we consider that the accretion flux is completely absorbed and re-radiated as a black body. For convenience, we define $T_{\text {irr }}$ as

$T_{\text {irr }}=\left(F_{\text {irr }}(t) / \sigma\right)^{1 / 4}$

where $F_{\text {irr }}$ is the irradiation flux and $\sigma$ is the Stefan-Boltzmann constant. The value of $F_{\text {irr }}$ (and consequently $T_{\text {irr }}$ ) is time dependent to take the temporal profile of the outburst into account. By $T_{\text {irr, } 0}$ we denote the maximum value of $T_{\text {irr }}$ reached during an outburst. For simplicity we take $T_{\text {irr, } 0}=10^{4} \mathrm{~K}$ for all models (see Smak 2004a). We use two outburst profiles, a "short" one lasting 60 orbital periods to model "standard" outbursts (note that we are not interested in complex behavior such as the outburst bimodality) and a "long" one lasting five times longer in order to model superoutburst of the SU UMa subtype (model 1), see Fig. 4. Because of the crudeness of the model, the exact shape and duration of our outburst are not of prime importance.

Irradiation produces a radiative isothermal layer whose column density depends on the irradiation temperature, on top of the convective envelope. Using simple vertical structure models for the secondary, we have computed the expected column density $\Sigma_{\text {irr }}$ of the isothermal layer when $T_{\text {irr }}=10^{4} \mathrm{~K}$. The results are given in Table 1. The initial column density is low (a few tens $\mathrm{g} \mathrm{cm}^{-2}$ ), because the irradiation flux is negligible, and it increases with time during an outburst. For the sake of simplicity, we do not consider the very initial stages of the outburst, and assume $\Sigma=\Sigma_{\text {irr }}\left(T=10^{4} \mathrm{~K}\right)$ as an initial condition. Since the precise value of $\Sigma$ affects only the thermal time, we want to obtain the correct order of magnitude of the cooling time scale for the gas heated at $T_{\text {irr }}=10^{4} \mathrm{~K}$.

The irradiation flux at a given point on the secondary surface enters as a heating term in the energy equation:

$F_{+}=\sigma\left(T_{\star}^{4}+\psi T_{\text {irr }}^{4}\right)$

where the intrinsic stellar flux is also included. The numerical factor $\psi$ has a space dependency to take oblique incidence and shading by the accretion disc on the secondary into account:

$\psi=($ n.u $) \times S\left(\alpha_{\mathrm{s}}\right)$. 


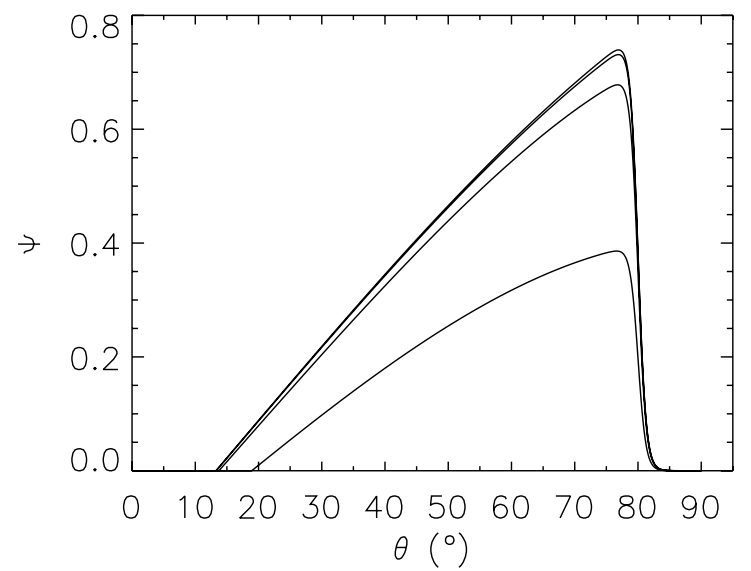

Fig. 5. Profile of $\psi$ along different meridians on the secondary: $\phi=0^{\circ}$ ( $L_{1}$ meridian), $\phi=5^{\circ}, \phi=15^{\circ}$ and $\phi=45^{\circ}$ (from top to bottom). The shadow boundary is located at $\theta=80^{\circ}$. Note that polar regions are not irradiated.

The first effect is accounted for by the $\boldsymbol{n} . \boldsymbol{u}$ term, where $\boldsymbol{n}$ is the local normal to the surface, and $\boldsymbol{u}$ is a unit vector pointing towards the primary, assumed to be the source of irradiation. The second effect is accounted for by the $S\left(\alpha_{\mathrm{s}}\right)$ term, where $\alpha_{\mathrm{s}}$ is the angle between the orbital plane and the line joining the primary center of mass and the running point on the secondary:

$\tan \alpha_{\mathrm{s}}=\frac{z}{\sqrt{(a-x)^{2}+y^{2}}}$

where $(x, y, z)$ are the Cartesian coordinates of the running point (in the same coordinate system as defined previously). We then assume that the disc has a fixed opening angle $\alpha_{\mathrm{d}}$ (see below) and we compute $S\left(\alpha_{\mathrm{s}}\right)$ by

$S\left(\alpha_{\mathrm{s}}\right)=\frac{1}{2}\left(1+\tanh \frac{\alpha_{\mathrm{s}}-\alpha_{\mathrm{d}}}{\Delta \alpha_{\mathrm{d}}}\right)$

where $\Delta \alpha_{\mathrm{d}}$ is an angular size controlling the thickness of the transition between the shaded part and the irradiated part, taken to be $\Delta \alpha_{\mathrm{d}}=0.5^{\circ}$. The hyperbolic tangent function enables a smooth transition between $\alpha_{\mathrm{s}}-\alpha_{\mathrm{d}} \ll \Delta \alpha_{\mathrm{d}}$ and $\alpha_{\mathrm{s}}-\alpha_{\mathrm{d}} \gg \Delta \alpha_{\mathrm{d}}$.

We use the numerical code from Hameury et al. (1998) to compute the vertical structure of the accretion disc during an outburst cycle. For each model, we determine the maximum value of the disc opening angle $\alpha_{\mathrm{d}}$. During an outburst, the disc opening is found to be $\alpha_{\mathrm{d}} \sim 4^{\circ}$ for model 1 and $\alpha_{\mathrm{d}} \sim 6^{\circ}$ for models 2 and 3 . In both cases this translates into a shadow boundary located at $\theta \sim 80^{\circ}$ when measured on the main meridian (the larger opening of the disc in models 2 and 3 is compensated by larger secondaries). The quiescent disc has a much lower opening that allows for a much smaller shaded region at the beginning of an outburst. We have checked that when the disc enters the outburst state, its opening increases very rapidly. Thus a good approximation is to suppose that the disc has its "outbursting thickness" since the very beginning of the outburst.

We use the mapping shown in Fig. 3 to project $\psi$ on the sphere of radius $R_{2}$ to obtain $\psi=\psi(\theta, \phi)$. Figure 5 shows $\psi$ along different meridians.

\subsection{Equations}

We now turn to the mathematical formulation of the equations. We use spherical coordinates $(\theta, \phi)(\theta$ is the colatitude and $\phi$ the longitude) to map the sphere of radius $r_{0}=R_{2}$. The metric properties of these coordinates are given by the metric tensor:

$\left(g_{i j}\right)=\left(\begin{array}{cc}r_{0}^{2} & 0 \\ 0 & r_{0}^{2} \sin ^{2} \theta\end{array}\right)$.

Here $g^{i j}$ denotes the components of the inverse of matrix (7) and $g$ denotes its determinant.

We use the following variables: $\Sigma$ is the gas surface density; $E, H=E+P$, and $P$ are the vertically integrated values of the energy density, enthalpy density, and pressure; $v_{\theta}, v_{\phi}$ are the velocity components. For convenience, we introduce the following notations:

- "Barred" quantities denote multiplication by $\sqrt{g}=r_{0}^{2} \sin \theta$. These quantities are corrected for the metric effects; for example, $\Sigma \mathrm{d} S=\Sigma \sqrt{g} \mathrm{~d} \theta \mathrm{d} \phi=\bar{\Sigma} \mathrm{d} \theta \mathrm{d} \phi$.

- "Tilted" velocity components are angular velocity components: $\tilde{v}_{\theta}=v_{\theta} / r_{0}=\dot{\theta}$ and $\tilde{v}_{\phi}=v_{\phi} /\left(r_{0} \sin \theta\right)=\dot{\phi}$.

With these notations, the Euler equations write as

$$
\begin{aligned}
\frac{\partial}{\partial t} \bar{\Sigma}+\frac{\partial}{\partial \theta} \bar{\Sigma} \tilde{v}_{\theta}+\frac{\partial}{\partial \phi} \bar{\Sigma} \tilde{v}_{\phi} & =0 \\
\frac{\partial}{\partial t} \bar{\Sigma} \tilde{v}_{\theta}+\frac{\partial}{\partial \theta} \bar{G}^{11}+\frac{\partial}{\partial \phi} \bar{G}^{12} & =f \bar{\Sigma} \tilde{v}_{\phi}+\cos \theta \sin \theta \bar{G}^{22} \\
\frac{\partial}{\partial t} \bar{\Sigma} \tilde{v}_{\phi}+\frac{\partial}{\partial \theta} \bar{G}^{21}+\frac{\partial}{\partial \phi} \bar{G}^{22} & =-f \bar{\Sigma} \tilde{v}_{\theta}-\frac{2}{\tan \theta} \bar{G}^{12} \\
\frac{\partial}{\partial t} \bar{E}+\frac{\partial}{\partial \theta} \bar{H} \tilde{v}_{\theta}+\frac{\partial}{\partial \phi} \bar{H} \tilde{v}_{\phi} & =\bar{F}_{+}-\bar{\sigma} T^{4}
\end{aligned}
$$

where $G^{i j}=\Sigma \tilde{v}^{i} \tilde{v}^{j}+P g^{i j}$ is the (symmetric) momentum flux tensor. A radiative cooling term is included on the right hand side of the energy equation in addition to the heating term (3).

The equations are written in conservative form and differ only slightly from the Cartesian case, with the inclusion of geometric source terms in the momentum equations (in addition to the Coriolis term) and with conservative variables $\left(\bar{\Sigma}, \bar{\Sigma} \tilde{v}_{\theta}, \bar{\Sigma} \tilde{v}_{\phi}, \bar{E}\right)$ that are not physical quantities. We also assume a perfect gas equation of state:

$P=\Sigma R_{\mathrm{g}} T$ and $E=\frac{1}{2} \Sigma v^{2}+\frac{1}{\gamma-1} P$

with an adiabatic index $\gamma=5 / 3$ and $R_{\mathrm{g}}=R / \mu, R$ being the perfect gas constant.

Note that we do not include viscosity. As in many astrophysical processes, the molecular viscosity is negligible, but turbulent viscosity could be significant. In a geostrophic state, Osaki \& Meyer (2003) show that viscosity is responsible for a drift across isobars, but the expected value of turbulent viscosity yields only a very low drift velocity.

\subsection{Simulation setup}

For each simulation, we start with an isothermal envelope with temperature $T_{\star}$ and surface density $\Sigma_{\text {irr }}$ (see Table 1$)$. The envelope is initially at rest; i.e., we neglect the quiescence steady surface flow feeding mass transfer. The order of magnitude of the quiescent flow speed is $\sim 0.01 c_{\mathrm{s}}$ (see Lubow \& Shu 1975), small compared to surface velocities found in outburst (see Sect. 4). Irradiation is then turned on with the temporal profile simulating an outburst or a superoutburst. Our simulations span a time length of $240 P_{\text {orb }}$. 


\section{The numerical code}

The Cartesian-like form of our equations enables us to directly apply any scheme developed for Cartesian coordinates. Here we use the TVD-MacCormack scheme (see Yee 1987) to solve Eqs. (8)-(11) for $\left(\bar{\Sigma}, \bar{\Sigma} \tilde{v}_{\theta}, \bar{\Sigma} \tilde{v}_{\phi}, \bar{E}\right)$. This scheme is a finite difference predictor-corrector scheme followed by a TVD step. This "Total Variation Diminishing" step ensures that non physical oscillations do not appear (see Hirsch 1990). The two-step nature of the scheme enables second-order accuracy in time, as well as a second-order discretization of the source terms. To obtain second order in space, the spatial discretization alternates between backward and forward differencing during the predictor and corrector steps. To avoid error accumulation, the order of forward/backward differencing is switched at each time-step. In steep gradient regions, the TVD step adds a diffusive flux locally altering the scheme order. The $\sin \theta \cos \theta$ term in Eq. (6) is discretized in such a way that the physical equilibrium $(P=$ cst. $)$ is also a solution to the discretized equation.

Due to the explicit character of the scheme, the numerical stability of the scheme is subject to the well-known CourantFriedrichs-Levy (CFL) condition; the time step is restricted by (see Hirsch 1990)

$$
\Delta t<\Delta t_{\max }=\frac{1}{\tilde{c}_{\theta}^{\max } / \Delta \theta+\tilde{c}_{\phi}^{\max } / \Delta \phi}
$$

where $\tilde{c}_{\theta}^{\max }=\max \left(c_{\mathrm{s}} / r_{0}+\left|\tilde{v}_{\theta}\right|\right)$ and $\tilde{c}_{\phi}^{\max }=\max \left(c_{\mathrm{s}} /\left(r_{0} \sin \theta\right)+\right.$ $\left.\left|\tilde{v}_{\phi}\right|\right)$. In our simulations we take $\Delta t=\lambda \Delta t_{\max }$ with $\lambda=0.95$. A simulation with $\lambda=0.4$ has been checked to give identical results.

We consider a physical domain $\theta_{0}<\theta<\pi-\theta_{0}$ and $-\pi<\phi<\pi$. We therefore exclude the two polar caps in order to avoid difficulties around the poles that are singular points in spherical coordinates. This is not a serious problem, as the poles are not irradiated (see Fig. 5). The grid-points accumulation occurring there would severely restrict the CFL condition. In the following, we choose $\theta_{0}=10^{\circ}$ and we have checked that this particular choice does not influence our results. We use an uniform grid with $151 \times 200$ grid points after checking the effect of numerical resolution for model 1 on $76 \times 100$ and $301 \times 400$ grids. As expected, the low-resolution run shows signs of enhanced numerical dissipation, and the high-resolution run shows a more turbulent behavior as compared to our standard resolution; but the main features and results presented here are not significantly modified by resolution effects (changes are less than $10 \%$ ). Note that the number of grid points in $\theta$ is odd in order to have a grid point at the equator. Periodic boundary conditions are used at $\phi= \pm \pi$, and free outflows conditions are considered at $\theta_{0}$ and $\pi-\theta_{0}$. We checked that this does not affect our results.

\section{Results}

In this section, the velocity, density, and temperature at $L_{1}$ refer to average quantities computed using a Gaussian kernel centered on $\left(\theta=90^{\circ}, \phi=0^{\circ}\right)$ with an FWHM equal to the radius of the cross section of the stream leaving the secondary (see Eq. (17) below).

\subsection{Model 1: the SU UMa class}

We first discuss normal outbursts. As expected, irradiation increases the surface temperature of the secondary and, as a result, pressure gradients form (see Fig. 6). In the presence of pressure gradients, the flow tends to reach geostrophism where the Coriolis force balances pressure gradients (i.e. velocity is along a constant pressure line). This dynamical phenomenon is known as "Rossby adjustment" or "geostrophic adjustment" (see Pedlosky 2003). Initially, vertical pressure gradients drive a vertical acceleration of the fluid, pushing it towards the equator; however, the Coriolis force quickly deflects the flow westwards, and this effect is stronger in the vicinity of $L_{1}$. This leads to an oscillatory pattern of the velocity field (see snapshots at $t=0.7 P_{\text {orb }}$ in Fig. 6) along the shadow boundary of the disc. This pattern drives the flow toward $L_{1}$. In addition, vertical pressure gradients appearing at high longitude, where the normal irradiation flux drops, are also adjusted; as a result, a clockwise circulation flow sets in. At $t=2.5 P_{\text {orb }}$ (see Fig. 6), the flow has reached geostrophism. The flow crosses isobars only in the close vicinity of $L_{1}$, as the Coriolis force is weaker there (see Sect. 2.1) and the flow speed greater. Note that the geostrophic state is significantly different from the one expected from the initial pressure gradients. This shows the complexity of the geostrophic adjustment process during which both the flow velocity and the pressure gradients vary, the irradiation flux being the only fixed quantity.

The overall situation can be depicted as irradiation driving a giant anti-cyclonic perturbation on each hemisphere of the secondary. Furthermore, the associated clockwise circulation goes through the $L_{1}$ point. At the same time, an equatorial jet traveling westwards forms at longitude $\phi \sim-60^{\circ}$, as well as a weaker one traveling eastwards at $\phi \sim 60^{\circ}$ (see snapshots at $t=2.5 P_{\text {orb }}$ in Fig. 6).

As time goes on, pressure gradients increase with irradiation and, as a result, the circulation strengthens (see Fig. 7 at $t=$ $30 P_{\text {orb }}$, the outburst maximum). The left panel of Fig. 8 shows the magnitude of the velocity at $L_{1}$. The maximum velocity is reached at $t=18 P_{\text {orb }}$ where $v_{\phi} \sim 0.6 c_{\mathrm{S}}$ (for symmetry $v_{\theta}=0$ ). The corresponding snapshot is shown in Fig. 6. The right panel of Fig. 8 shows the evolution of the surface density at $L_{1}$. In the early stage of the outburst, the surface density increases by a few percent as matter is initially being driven toward the equator. Later on, it decreases temporarily by a few percent due to the strong surface flow through $L_{1}$ and as a consequence of mass conservation.

Snapshots at different times (see Figs. 6, 7) show that the anti-cyclonic perturbation slowly drifts westward. This is a natural consequence of its clockwise circulation and it shows that no steady state can ever be reached. As a consequence, the circulation flow initially transiting by the $L_{1}$ point moves away from $L_{1}$ (see left panel of Fig. 8 for $t<50 P_{\text {orb }}$ ). Note also that the temperature distribution at the surface of the secondary is not affected much by the flow, as would be the case if heat transport by advection were important. This is an important point that will be discussed in more detail later on.

The subsequent increase in the velocity at $L_{1}$ in Fig. 8 (left panel, at $t \sim 70 P_{\text {orb }}$ ) is due to the transit of the westwards jet. At the outburst end, the residual circulation flow slowly decays (due to numerical dissipation) on a shorter time scale than the time interval between outbursts; physical viscosity (e.g. due to turbulence) would bring the velocity field to zero before the next outburst starts.

Numerical simulations of a superoutburst show the same features during the initial phase, as expected. Even after $t=$ $30 P_{\text {orb }}$, when a normal outburst would have started to decline, the surface flow is not very different in the normal and superoutburst cases, see Fig. 7. Due to the maintained irradiation, the strength of the surface flow does not decay as it does in 

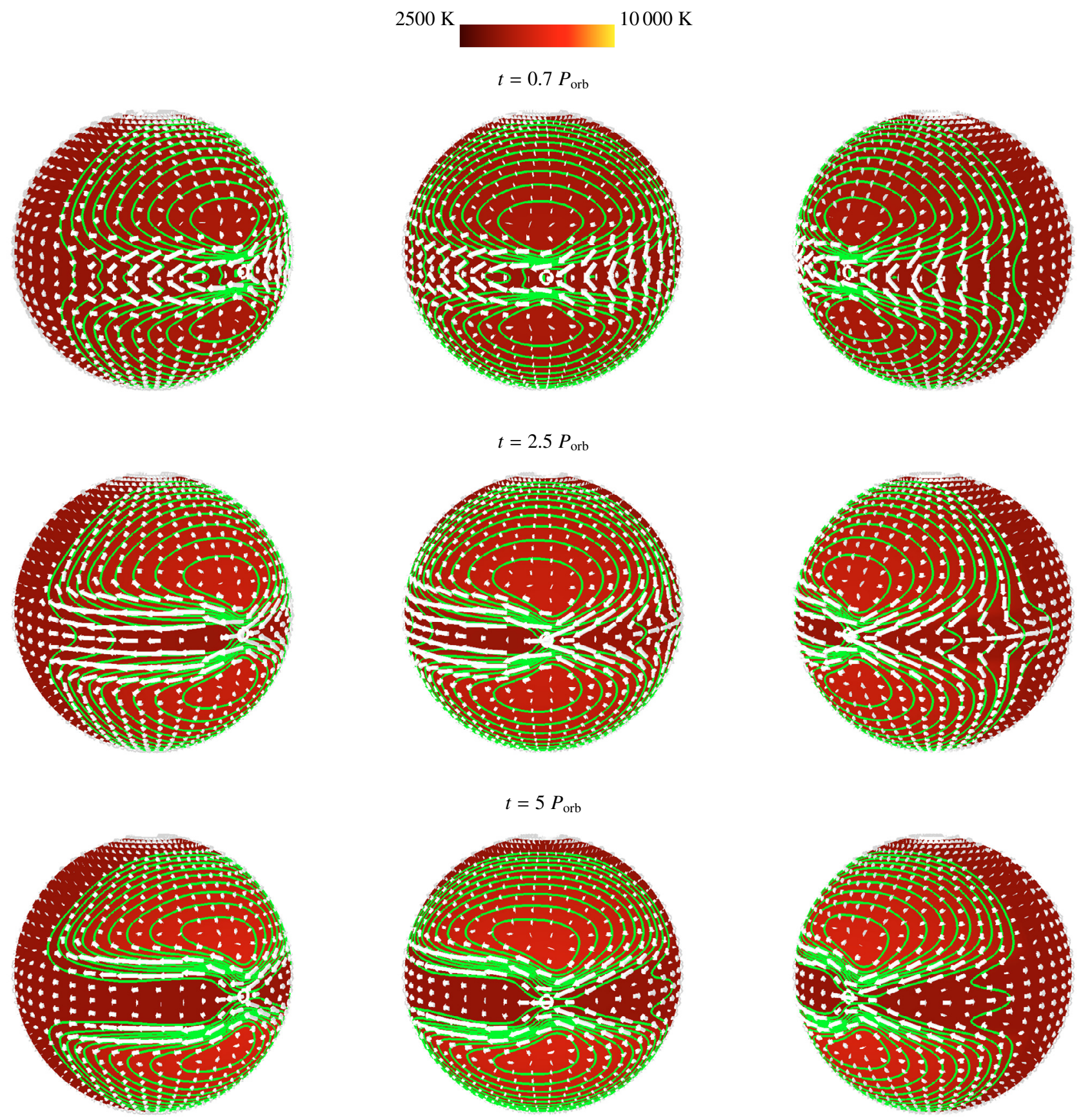

Fig. 6. Snapshots at $t=0.7,2.5,5 P_{\text {orb }}$. Each snapshot shows the temperature field in color gradient (see the color legend), isobars, and the velocity field. The central panel shows the secondary face on; in the left and right panels, it is rotated by $+/-30$ degrees. The $L_{1}$ region is marked by a circle whose surface is equal to the stream cross section (see Eq. (17)). For the sake of figure readability, the magnitude of the velocity field has been multiplied by 3 at $t=0.7 P_{\text {orb }}$ and by 2 at $t=2.5 P_{\text {orb }}$.

the normal outburst case. However, the anti-cyclonic perturbation drifts westward in the same way as it does in the normal outburst case, also suppressing the flow transiting by $L_{1}$ (see left panel of Fig. 8). The subsequent evolution shows a turbulent behavior that slowly decays when the superoutburst ends. The surface density at $L_{1}$ (see right panel of Fig. 8) is increased by at most $20 \%$ during a superoutburst.

To compare our results with those from Smak (2004a), we now follow the trajectories of test particles distributed in the atmosphere and initially at rest. Figure 9 (left panel) shows a few example of such trajectories. Each one lasts for
40 orbital periods. This figure shows that particles initially at longitude $\phi \sim 40^{\circ}-50^{\circ}$ reach the close vicinity of $L_{1}$. The arrival time in the $L_{1}$ region can be obtained from the right panel of Fig. 9, which shows the time evolution of each particle colatitude. Figure 10 shows the temperature of each particle vs. colatitude. The particles are heated during their trajectory towards $L_{1}$, and they cool down very quickly once they enter the shaded region. As a consequence, the temperature of the $L_{1}$ point remains very close to its quiescent value $(2500 \mathrm{~K})$, in contrast to Smak (2004a). The reason for this is that the cooling time scale is shorter than the transit time towards $L_{1}$ : as shown in the right 

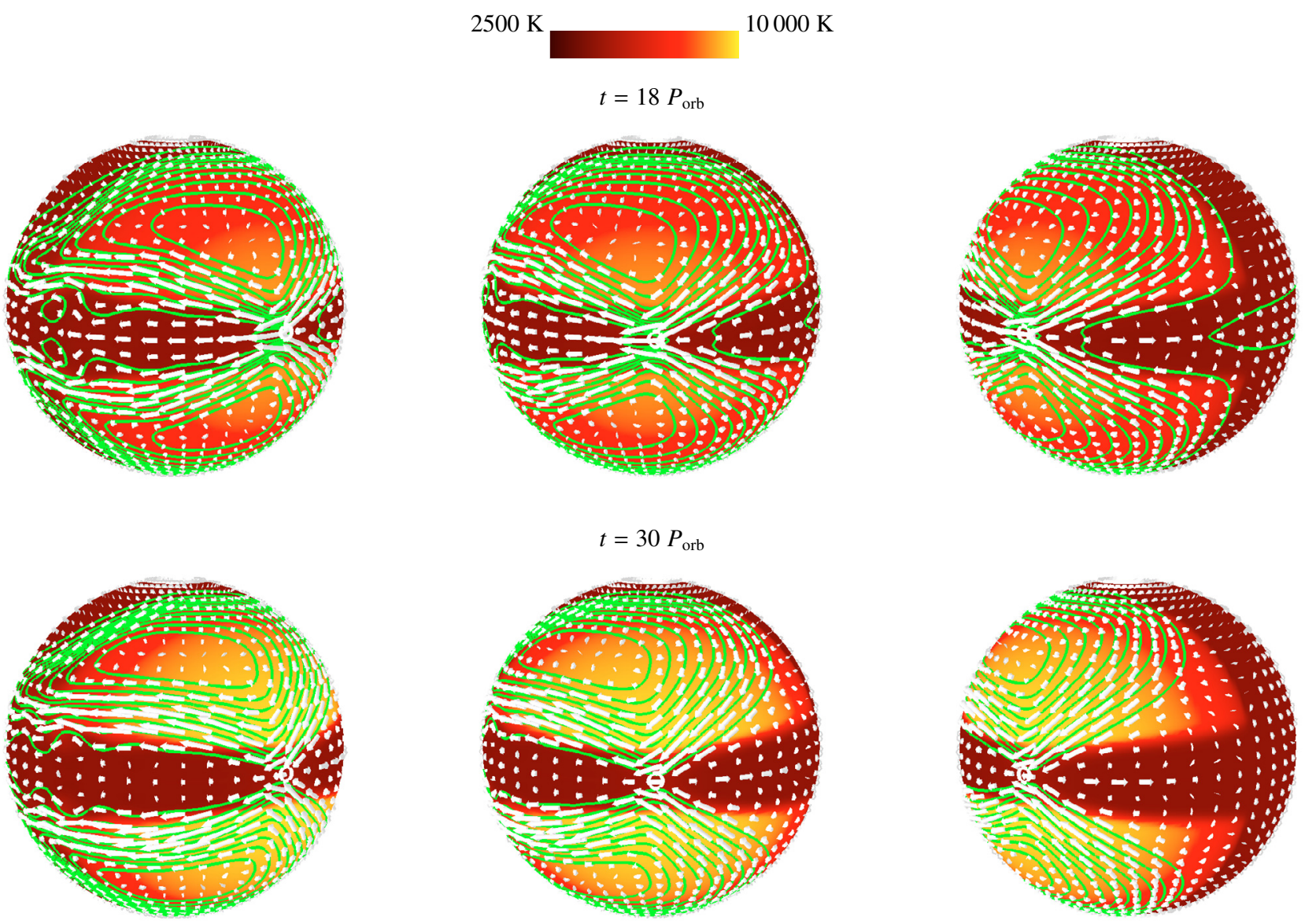

$t=30 P_{\text {orb }}$
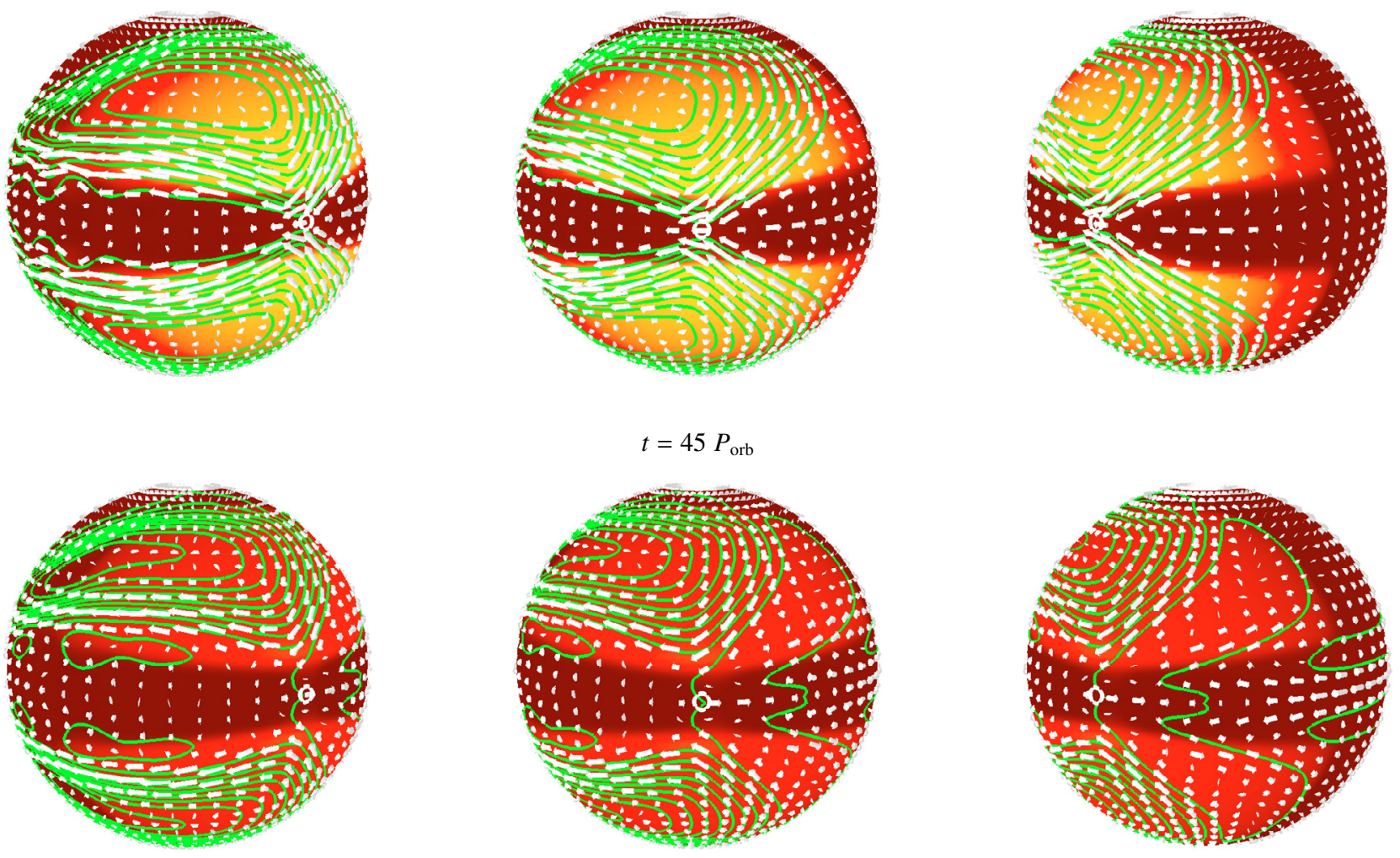

$t=45 P_{\text {orb }}$ during a superoutburst
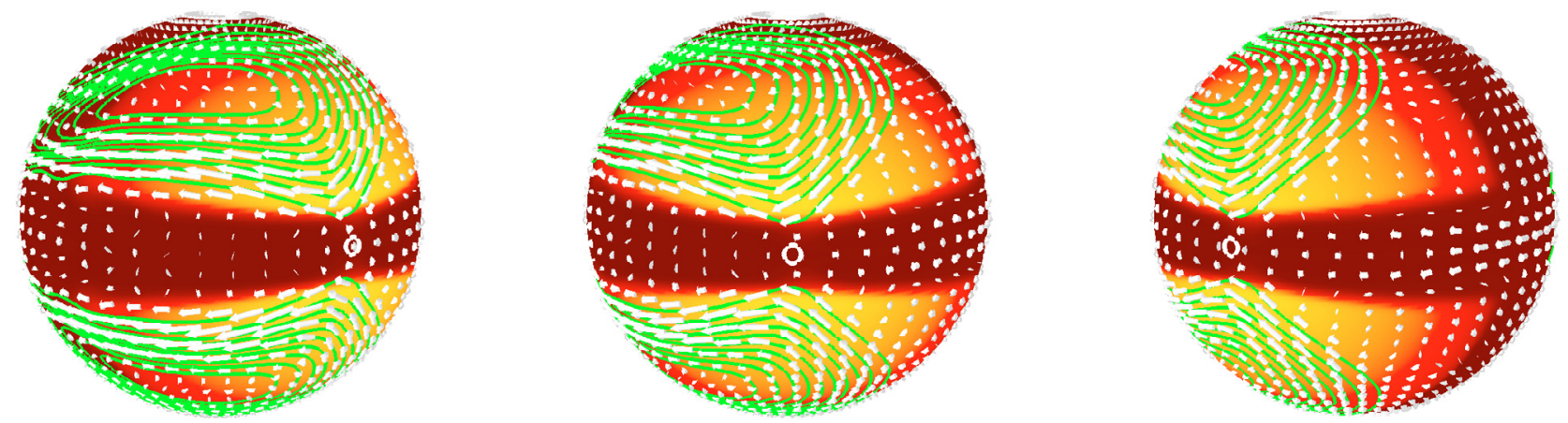

Fig. 7. Snapshots at $t=18,30,45 P_{\text {orb }}$. Each snapshot shows the temperature field in color gradient (see the color legend), isobars, and the velocity field. 

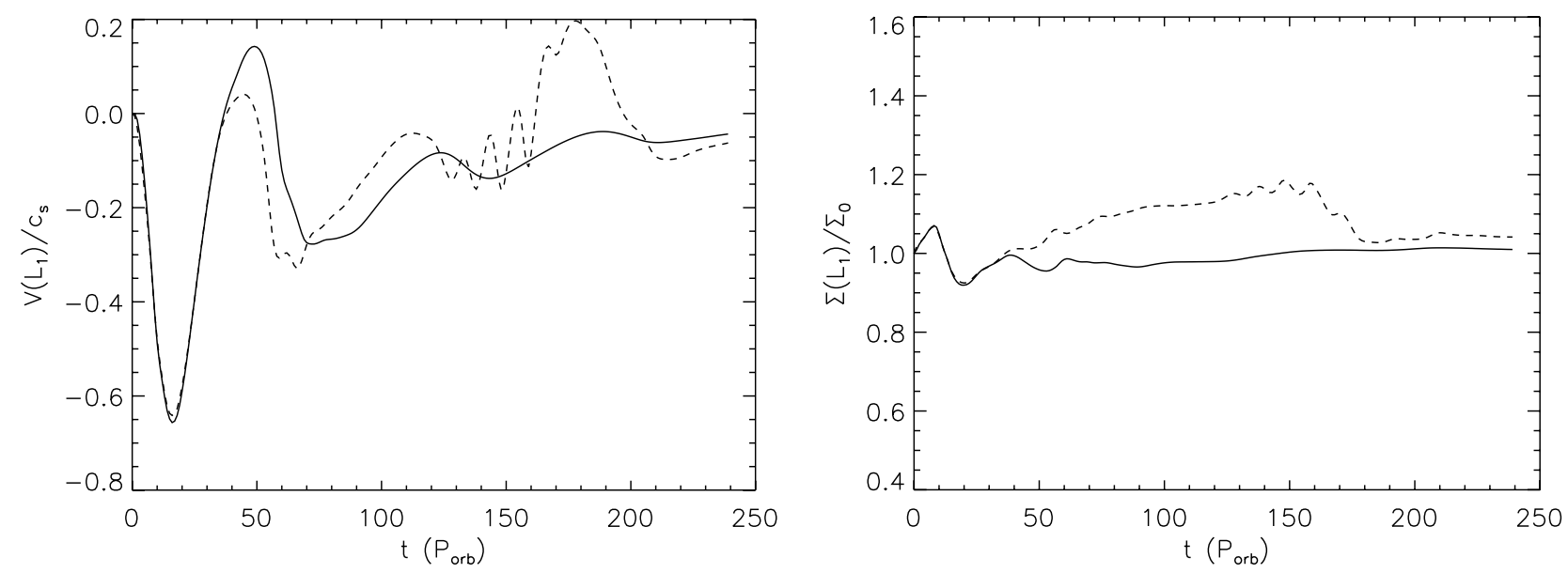

Fig. 8. Left panel: evolution of the velocity at $L_{1}$ normalized by the isothermal sound speed (computed with the temperature at $\left.L_{1}\right)$ during an outburst (solid line) and a superoutburst (dashed line). The maximum velocity is reached at $t=18 P_{\text {orb }}$ (see Fig. 7). Right panel: evolution of the surface density at the $L_{1}$ point normalized by its initial value surface density during an outburst (solid line) and a superoutburst (dashed line).
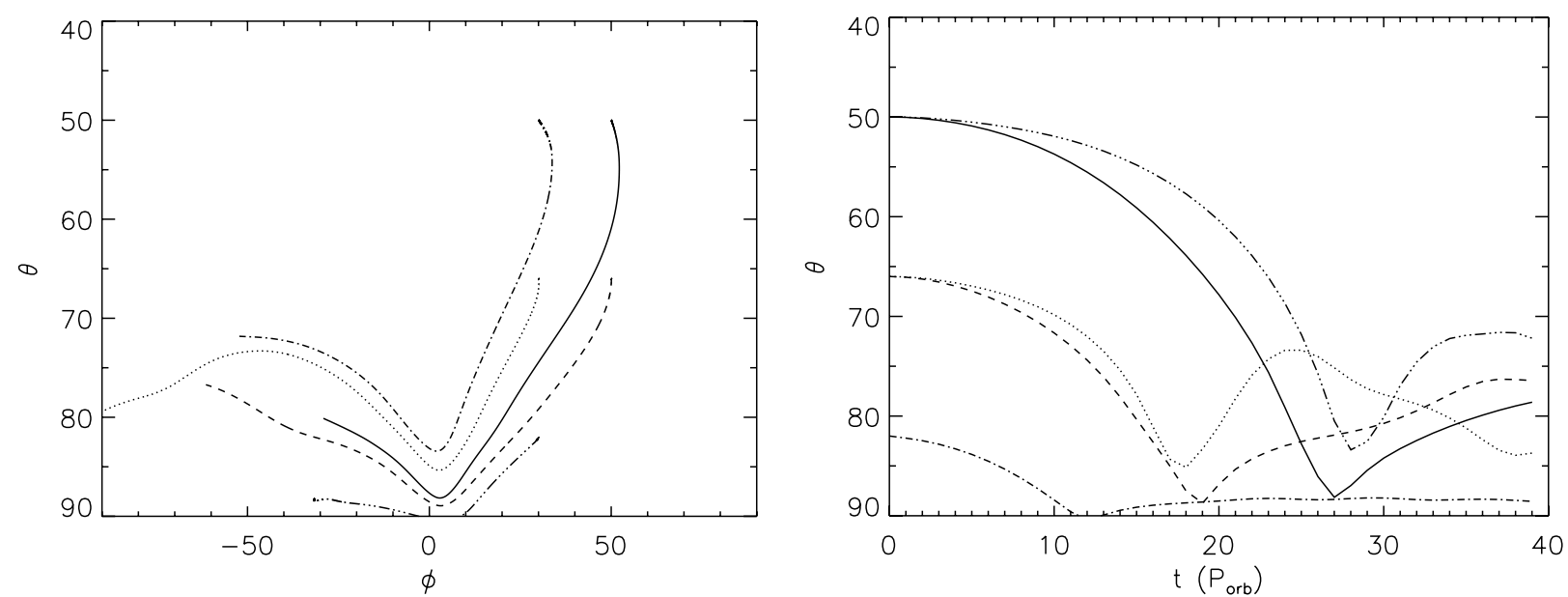

Fig. 9. Left panel: particle pathlines from $t=0$ to $t=40 P_{\text {orb }}$. Right panel: time evolution of the colatitude of each particles. The shadow boundary is at $\theta=80^{\circ}$.

panel of Fig. 9, the time scale for crossing the shadow boundary is on the order of a few orbital periods. The cooling time scale is

$\tau_{\mathrm{cool}}=\frac{\Sigma c_{v} T}{\sigma T^{4}}$

where $c_{v}$ is the specific heat capacity. Thus $\tau_{\text {cool }} \propto \Sigma T^{-3}$ with a very strong temperature dependence. For an initial value $\Sigma=$ $350 \mathrm{~g} \mathrm{~cm}^{-2}, \tau_{\text {cool }}=9 P_{\text {orb }}$ for $T=2500 \mathrm{~K}$, but $\tau_{\text {cool }}=0.15 P_{\text {orb }}$ for $T=10^{4} \mathrm{~K}$. Consequently, as materials heated at $T \sim 10^{4} \mathrm{~K}$ enter the shaded region, their very rapid cooling is unavoidable. A more elaborate computation of a $T^{4}$ cooling shows that, after a few cooling time scales, the gas temperature is close to the ambient value. The preceding argument holds even better when one corrects for the Roche geometry (see Sect. 2): real distances are about $30 \%$ larger. The cooling of the gas is so efficient that this conclusion does not depend much on the thickness of the shaded region (see discussion at the end of Sect. 2.2) or on the exact value of $\Sigma_{\text {irr }}$, since it would require unrealistically high values of $\Sigma$ for $\tau_{\text {cool }}$ to be on the order of the crossing time scale.

\subsection{Models 2 and 3: U Gem and Z Cam classes}

Numerical simulations with parameters corresponding to U Gem (model 2) and Z Cam (model 3) show very similar features

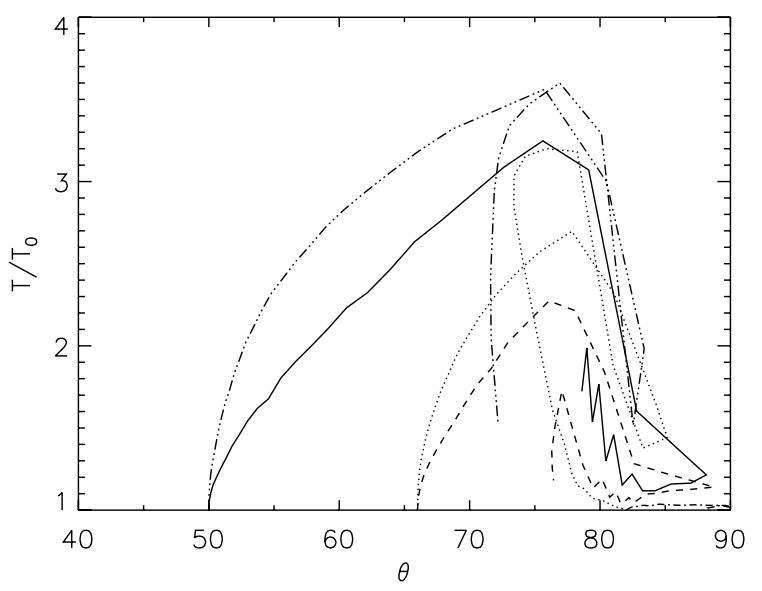

Fig. 10. Temperature vs. colatitude for each particle $\left(T_{0}=2500 \mathrm{~K}\right.$, the shadow boundary is at $\theta=80^{\circ}$ ). Particles cool very rapidly as they enter the shaded region.

to the SU UMa case. In particular, the time needed for particles to cross the shadow boundary is close to what was previously found, namely a few orbital periods while the cooling time is unchanged. For the parameters of models 2 

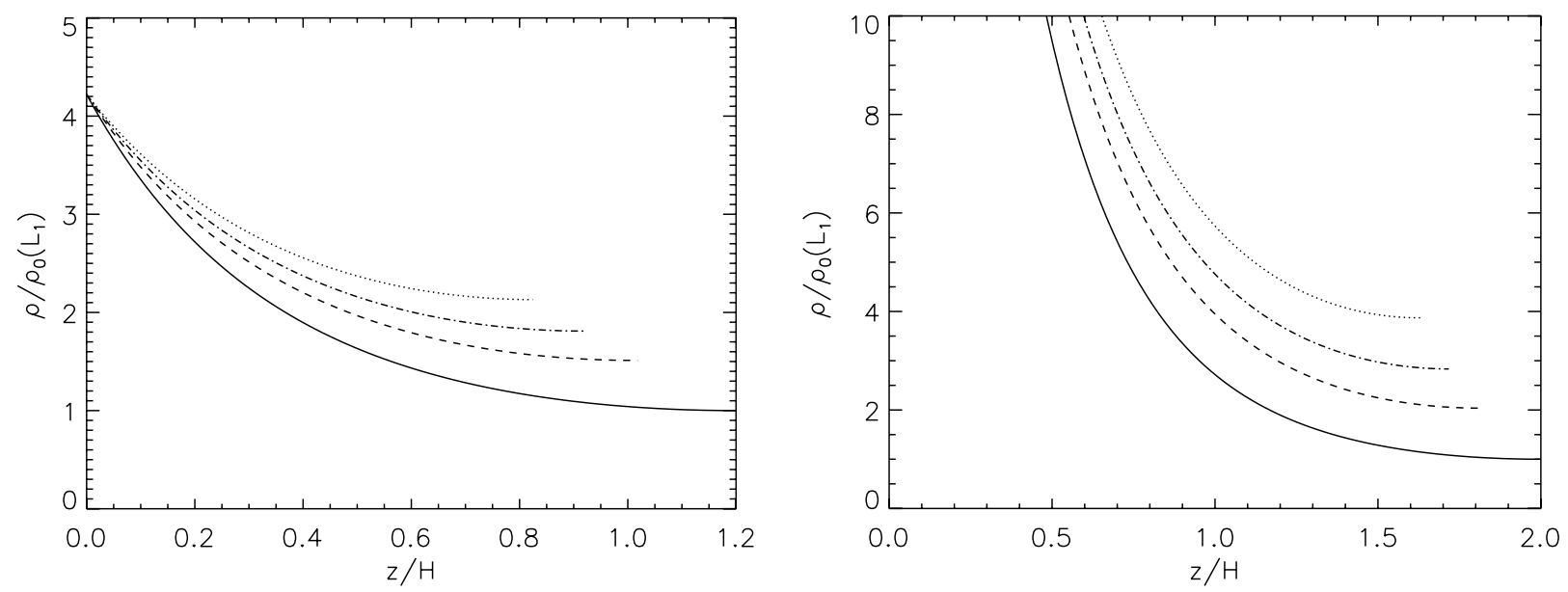

Fig. 11. Vertical profile of the density $\rho$ in the isothermal envelope when $v_{\theta}=0, v_{\theta}=0.5 c_{\mathrm{s}}, v_{\theta}=0.75 c_{\mathrm{s}}$, and $v_{\theta}=c_{\mathrm{s}}$ (from bottom to top). The left panel corresponds to $z_{L_{1}}=1.2 \mathrm{H}$ and right panel corresponds to $z_{L_{1}}=2 \mathrm{H}$. The density is normalized by the value of the density at $L_{1}$ when $v_{\|}=0$.

and $3 \tau_{\text {cool }} \sim 0.02-0.03 P_{\text {orb }}$ (due to larger orbital periods), thus showing that the above conclusion also applies here.

\subsection{Mass transfer enhancement?}

We now discuss the implications of our numerical results on a possible mass transfer enhancement. The mass transfer rate is given by (see Lubow \& Shu 1975; Meyer \& Meyer-Hofmeister 1983):

$\dot{M}=Q \rho\left(L_{1}\right) c_{\mathrm{s}}$

where $c_{\mathrm{s}}$ is the isothermal sound speed

$c_{\mathrm{s}}=\sqrt{R_{\mathrm{g}} T\left(L_{1}\right)}$

and $Q$ is the cross section of the stream (see Meyer \& Meyer-Hofmeister 1983):

$Q=\frac{2 \pi}{k}\left(\frac{c_{\mathrm{s}}}{\Omega}\right)^{2}$

where $k$ is a constant that depends only slightly on the mass ratio $q$.

The factor $Q c_{\mathrm{s}}$ yields a $T\left(L_{1}\right)^{3 / 2}$ dependence of the mass transfer on the temperature at $L_{1}$. If we further assume that vertical hydrostatic equilibrium prevails, $\rho\left(L_{1}\right)$ also depends on $T\left(L_{1}\right)$ and on $\Sigma\left(L_{1}\right)$. Our results have shown that $T\left(L_{1}\right)$ is almost unaffected by irradiation and heat transport. An increase in $\Sigma\left(L_{1}\right)$ by a few percent leads to an increase in $\dot{M}$ by the same amount and is therefore inefficient for increasing the mass transfer rate (see right panel of Fig. 8). However, beneath $L_{1}$ the Coriolis force pushes the gas in the vertical direction, which could change the vertical distribution of the mass density. Including the Coriolis force in the hydrostatic balance gives

$\frac{\partial P}{\partial z}=-\rho g+\rho 2 \Omega v_{\theta}=-\rho g_{\mathrm{eff}}$

where the $z$ axis now refers to the local vertical axis with the origin taken at the base of the isothermal atmosphere, $g$ is the Roche gravity and $v_{\|}$is the surface velocity of the gas.

Due to the Coriolis force, the location where the effective gravity vanishes can be lower than the $L_{1}$ point. In this case, one should replace $\rho\left(L_{1}\right)$ in Eq. (15) by the density at the point where the effective gravity vanishes. When $v_{\|}=0$, Eq. (18) can be solved by expanding $g$ to the first order around $z_{L_{1}}$, the height of $L_{1}$ (see e.g. Lubow \& Shu 1975), giving

$\rho_{0}(z)=\rho_{0}\left(L_{1}\right) \exp \left(\left(z-z_{L_{1}}\right)^{2} / H^{2}\right)$

where $H=c_{\mathrm{s}} /(\Omega \sqrt{A+1 / 2})$ is the vertical scale height of the isothermal envelope. The numerical factor $A$ is given in Lubow \& Shu (1975).

Since we do not known the vertical profile of $v_{\|}$, we solve Eq. (18) with $v_{\theta}=0.5,0.75,1.0 c_{\mathrm{s}}$, and the results are shown in Fig. 11. The mass transfer enhancement is directly given by the increase in the density. Results depend on the ratio $z_{L_{1}} / H$, which has to be determined by computing the vertical structure of the envelope. This is beyond the scope of the present paper, but we consider two cases in Fig. 11: $z_{L_{1}} / H=1.2$ and $z_{L_{1}} / H=2$.

It should be noted that, as the velocity reaches a fraction of the sound speed, it is possible that the hydrostatic equilibrium assumption breaks in the whole vertical extent of the envelope. A detailed analysis would require 3D simulations, beyond the scope of this paper. However, the main conclusion that the cooling time is much shorter than the crossing time of the disc shadow will not change.

Finally, note that a mass transfer enhancement due to the strong surface flow, whatever the mechanism at play, would be restricted to the rise of the outburst or superoutburst (see left panel of Fig. 8 for $t<30 P_{\text {orb }}$ ).

\section{Conclusion}

We have numerically investigated the surface flow of the irradiated secondary star in dwarf novae, using a simple model for both the geometry and irradiation of the secondary star. Our numerical simulations can be viewed as large-scale meteorological simulations of irradiated secondary stars: irradiation triggers a large anti-cyclonic perturbation (i.e. high pressure perturbation) with the associated clockwise circulation due to the Coriolis force. An important result of our numerical simulations is that the Roche geometry naturally leads to a circulation flow transiting by $L_{1}$, contrary to naive expectations. This provides a way to transport matter/heat in the vicinity of $L_{1}$, where matter leaves the secondary. We have also shown that it is necessary to solve the full time-dependent problem in order to find the dynamics of the geostrophic adjustment process. The flow does not reach the geostrophic state without modifying the background pressure 
gradients (as is implicitly done in Osaki \& Meyer 2003, 2004). It is also important to note that no steady state can be reached on the time scale of an outburst or a superoutburst.

We modeled the irradiation and radiative cooling of the envelope of the secondary in a very crude way, thus avoiding the necessity of solving the vertical structure of the atmosphere. In our model, radiative cooling occurs on a very short time scale, much shorter than the crossing time scale of the shaded region. There is thus no efficient heat transport in the vicinity of $L_{1}$. It is unlikely that this conclusion will change in a $3 \mathrm{D}$ approach that would also include a more realistic treatment of radiative transfer. As a consequence, if hydrostatic equilibrium prevails, the only effect that could lead to a moderate enhancement of mass transfer is the vertical Coriolis force. It could be possible, however, that hydrostatic equilibrium breaks down as the magnitude of the surface flow approaches the sound speed. A 3D approach would then be necessary to determine the mass flux leaving the secondary. Note that we have not considered the magnetic field of the secondary here. For the expected range of intensity, the magnetic field is unlikely to dominate the gas dynamics, but its influence on the flow could be of some importance. A correct implementation of the magnetic field configuration would be very difficult, however, and is far beyond the scope of the paper.

Apart from the transport mechanism investigated here, any other mechanisms that could directly heat the $L_{1}$ point would also contribute to an increase in the mass transfer rate. For example, the direct heating of the $L_{1}$ point by the rim of the accretion disc or by the scattering of the accretion luminosity by outflowing matter are currently under investigation to check if the heating of $L_{1}$ could be efficient. We leave this for a forthcoming paper.

Even if the circulation flow found here were to lead to a significant increase of the mass transfer rate, this effect would not last longer than 10-20 orbital periods, as the anti-cyclonic perturbation moves rapidly westwards, quenching the flow through $L_{1}$ on a time scale comparable to the rise time of the outburst. We would expect in this case no differences between outbursts and superoutbursts.

Finally, it could be tempting to apply these results to soft X-ray transients (see e.g. Chen et al. 1997, for a review). In these systems the irradiation flux is larger than in DN by a factor of order $10^{3}$ and consequently $T_{\text {irr }} \sim 5 \times 10^{4} \mathrm{~K}$. Such a strong irradiation raises very important pressure gradients, and one could expect supersonic velocities. A numerical investigation of this case would need a more robust numerical code.

\section{References}

Cannizzo, J. K. 1993, in Accretion Disks in Compact Stellar Systems, World Scientific

Chen, W., Schrader, C. R., \& Livio, M. 1997, ApJ, 491, 312

Hameury, J.-M. 2000, New Astron. Rev., 44, 15

Hameury, J.-M., Menou, K., Dubus, G., Lasota, J. P., \& Hure, J. M. 1998, MNRAS, 298, 1048

Hameury, J. M., Lasota, J. P., \& Warner, B. 2000, A\&A, 353, 244

Hirsch, C. 1990, in Numerical Computation Of Internal And External Flows, vol. 2 (John Wiley \& Sons)

Kopal, Z. 1969, Ap\&SS, 5, 360

Lasota, J.-P. 2001, New Astron. Rev., 45, 449

Meyer, F., \& Meyer-Hofmeister, E. 1983, A\&A, 121, 29

Lubow, S. H., \& Shu, F. H. 1975, ApJ, 198, 383

Osaki, Y., \& Meyer, F. 2003, A\&A, 401, 325

Osaki, Y., \& Meyer, F. 2004, A\&A, 428, 17

Pedlosky, J. 1982, Geophysical Fluid Dynamics (Springer)

Pedlosky, J. 2003, Waves in the Ocean and Atmosphere (Springer)

Sarna, M. J. 1990, A\&A, 239, 163

Smak, J. 1995, Acta Astron., 45, 355

Smak, J. 1999, Acta Astron., 49, 391

Smak, J. 2000, New Astron. Rev., 44, 171

Smak, J. 2004a, Acta Astron., 54, 181

Smak, J. 2004b, Acta Astron., 54, 221

Smak, J. 2004c, Acta Astron., 54, 429

Vogt, N. 1985, A\&A, 118, 95

Walter, W. H., Van Paradijs, J., \& Van Den Heuvel, E. P. 1995, X-Ray binaries (Cambridge University Press)

Warner, B. 1995, Catacysmic Variable stars (Cambridge University Press)

Yee, H. C. 1987, NASA Technical Memorandum 89464 